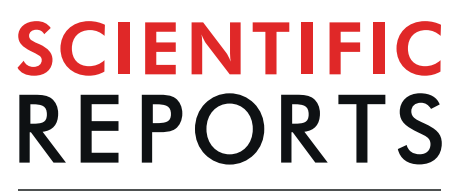

Received: 19 June 2019

Accepted: 15 September 2019

Published online: 01 October 2019

\section{Effect of Water Avoidance Stress on serum and urinary NGF levels in rats: diagnostic and therapeutic implications for BPS/IC patients}

\author{
Bruno Dias $^{1,2}$, Paula Serrão ${ }^{3,4}$, Francisco $\operatorname{Cruz}^{2,5,6,7}$ \& Ana Charrua $\mathbb{D}^{1,6,7}$
}

Nerve growth factor (NGF) is thought to play a key role in chronic pain felt by bladder pain syndrome/ interstitial cystitis (BPS/IC) patients by activating its high affinity receptor tropomyosin-related kinase subtype $A$ (Trk $A$ ). Whether this pathway is also involved in the aggravation of pain sensation during stress events was here investigated. The levels of plasmatic NGF were increased in rats submitted to Water Avoidance Stress test (WAS), compared to controls. The administration of the alpha1A adrenoceptors blocker silodosin prevented the increase of plasmatic NGF. Urinary NGF levels were also moderately increased in animals submitted to WAS. WAS increased pain behaviour score, lowered abdominal mechanical pain threshold and increase voiding bladder reflex activity. These changes were prevented by the administration of TrkA antagonist GW441756. These findings prompt the use of plasmatic NGF as diagnosis tool for chronic visceral painful conditions and opens therapeutic opportunities for TrkA receptors antagonist/NGF sequestration.

NGF is a master modulator of neural plasticity in particular of nociceptive peptidergic neurons, by recruiting, phosphorylating and activating the tropomyosin-related kinase subtype A (TrkA) receptor ${ }^{1}$. Multiple studies have described that the urine of IC/BPS patients and of rodent models of this human disease have elevated levels of nerve growth factor (NGF) incompared to controls ${ }^{2-6}$. NGF is also elevated in the serum of these patients, although this fact has been infrequently reported ${ }^{7}$. In a recent pooled analysis from 3 small clinical trials, women with BPS/IC and with symptoms suggesting the concomitant presence of non-urological somatic painful syndromes are those that benefit more from NGF sequestration treatment ${ }^{8}$. Therefore, it is not surprising that the sequestration of NGF by its highly specific humanized monoclonal antibody is under investigation to manage bladder pain in BPS/IC patients.

The high levels of NGF observed in BPS/IC patients have been attributed to the excess of synthesis and release of the neurotrophin from the bladder urothelium and detrusor smooth muscle cells ${ }^{9,10}$. In this hypothesis, the high urinary levels of NGF results from its diffusion from the bladder wall into the urine and the NGF serum levels would be the consequence of its diffusion from the bladder tissue into the blood vessels.

Typically, BPS/IC patients report an increase in bladder pain intensity during stressful events ${ }^{11}$. If such events cause an increase of NGF in the urine or in the serum, therefore contributing to pain exacerbation, it is presently unknown. Nevertheless, this information can be highly relevant in order to determine if patients under stressful conditions also benefit from NGF sequestering strategy. It may be recalled that BPS/IC patients also show an increase of noradrenaline (NA) levels in the plasma and in the urine ${ }^{12}$. Excess of noradrenaline may lead by itself to pain enhancement by activating the numerous alpha-adrenoceptors expressed by nociceptive sensory fibers ${ }^{13}$.

\footnotetext{
${ }_{1}^{1}$ Departamento de Biomedicina - Unidade de Biologia Experimental, Faculdade de Medicina da Universidade do Porto, Porto, Portugal. ${ }^{2}$ Departamento de Urologia, Centro Hospitalar de São João, Porto, Portugal. ${ }^{3}$ Departamento de Biomedicina - Unidade de Farmacêutica e Terapêutica, Faculdade de Medicina da Universidade do Porto, Porto, Portugal. ${ }^{4}$ MedInUP - Center for Drug Discovery and Innovative Medicines, University Porto, Porto, Portugal. ${ }^{5}$ Serviço de Urologia Faculdade de Medicina da Universidade do Porto, Porto, Portugal. ${ }^{6} \mathrm{i} 3 \mathrm{~S}$ - Instituto de Investigação e Inovação em Saúde, Universidade do Porto, Porto, Portugal. ${ }^{7}$ IBMC - Instituto de Biologia Molecular e Celular, Universidade do Porto, Porto, Portugal. Correspondence and requests for materials should be addressed to A.C. (email: anacharr@gmail.com)
} 
A

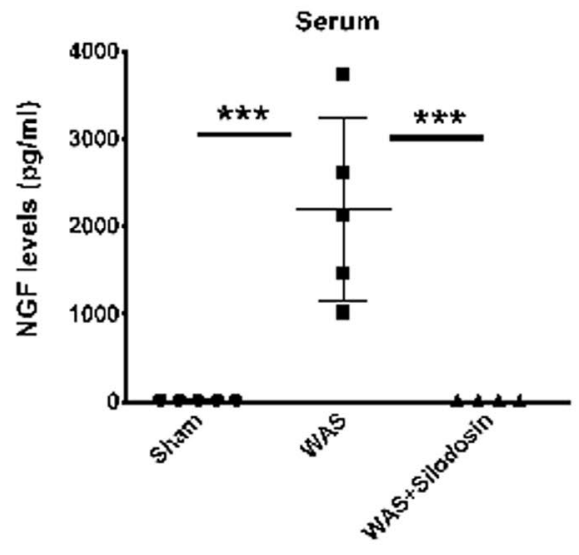

B

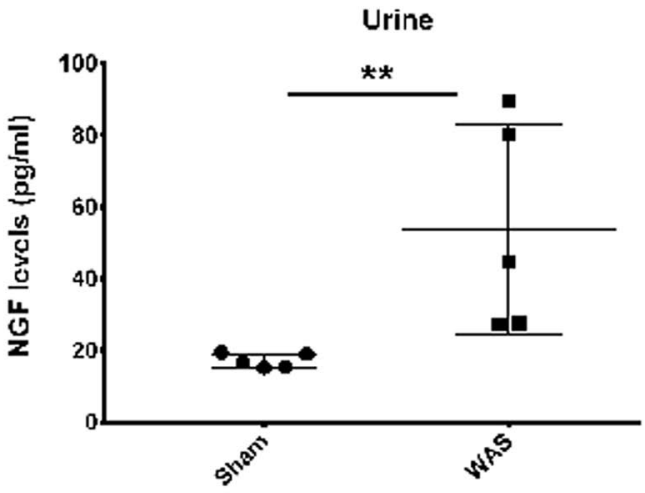

C

Bladder explant medium - 24h

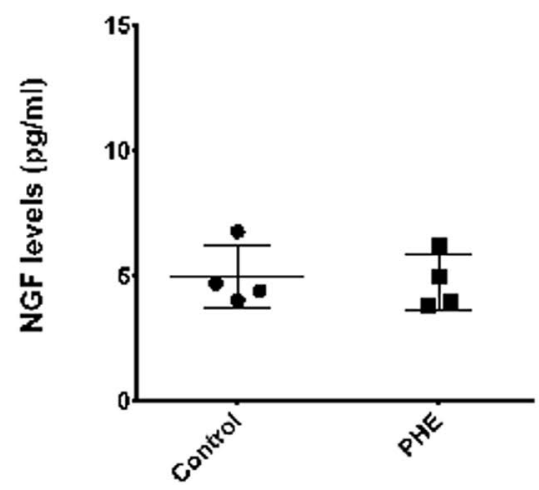

Figure 1. Scatter graph showing the NGF levels $(\mathrm{pg} / \mathrm{ml})$ in (A) serum of sham, WAS and WAS + Silodosin groups; (B) urine of sham and WAS groups; (C) bladder explant medium after $24 \mathrm{~h}$ of exposition to medium (control) and phenylephrine (PHE). $* * \mathrm{p}<0.01 ; * * \mathrm{p}<0.001$.

To clarify the role of NGF in pain exacerbation during stressful conditions, in the present work we used an animal model of chronic stress, the Water Avoidance Stress (WAS), a rat model that is known to course with intense, prolonged bladder pain. We investigated the serum and urinary levels of NGF and the effect of the blockade of TrkA, the high affinity NGF receptor, in bladder pain and bladder function.

\section{Results}

NGF levels determination by ELISA. Plasmatic levels of NGF on sham group were $19.18 \pm 0.47 \mathrm{pg} / \mathrm{ml}$ (Fig. 1A). WAS induced a marked increase in plasmatic NGF levels to $2194.17 \pm 942.73 \mathrm{pg} / \mathrm{ml}$ (Fig. 1A; ANOVA followed by Tukey's multiple comparison test: $\mathrm{p}<0.0001)$. Concerning urinary NGF, animals from sham group presented $17.17 \pm 1.73 \mathrm{pg} / \mathrm{ml}$ (Fig. 1B). In the WAS group, there was a moderate increase of the urinary NGF to $53.91 \pm 26.26 \mathrm{pg} / \mathrm{ml}$ (Fig. 1B; unpaired T-test: $\mathrm{p}=0.0235$ ).

The administration of the apha1 A antagonist silodosin during WAS induction prevented the increase in serum NGF levels $(17.50 \pm 0.53 \mathrm{pg} / \mathrm{ml}$; ANOVA followed by Tukey's multiple comparison test: $\mathrm{p}=0.0001$ compared to WAS and $\mathrm{p}=1$ compared with sham). 
A

Test A - WAS group

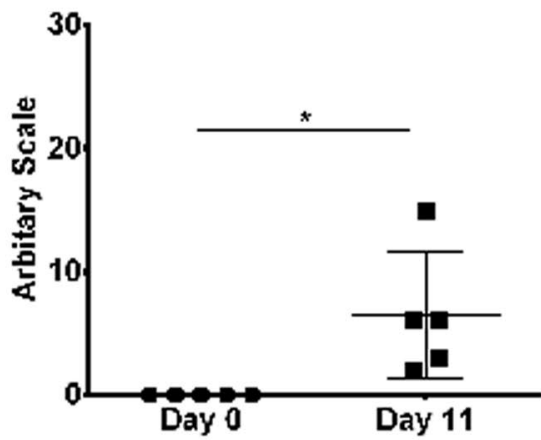

C

Test A .

WAS + GW441756 group

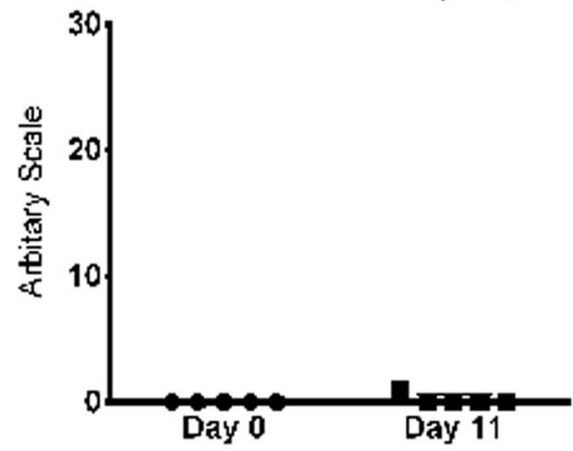

E Von Frey Test - Was group

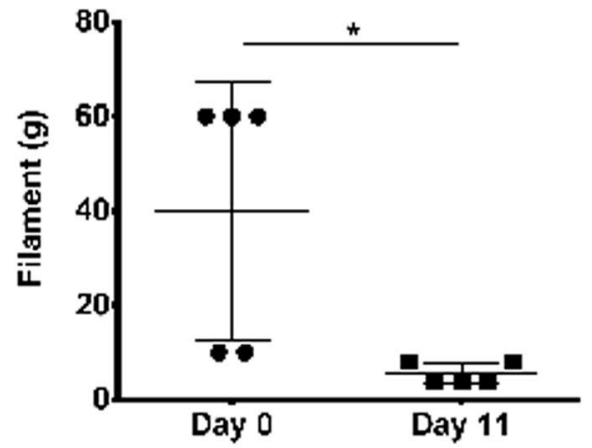

B Test B - WAS group

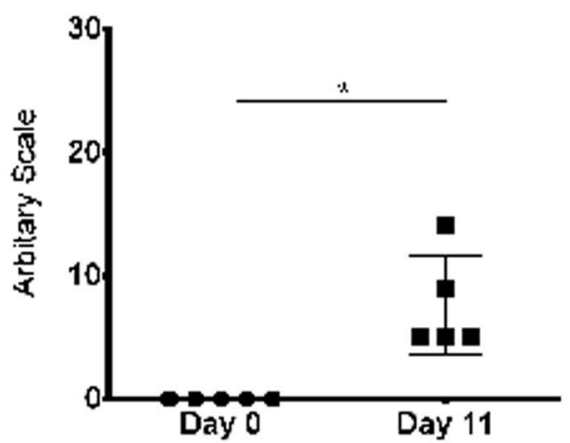

$\mathbf{D}$

Test B WAS + GW441756 group
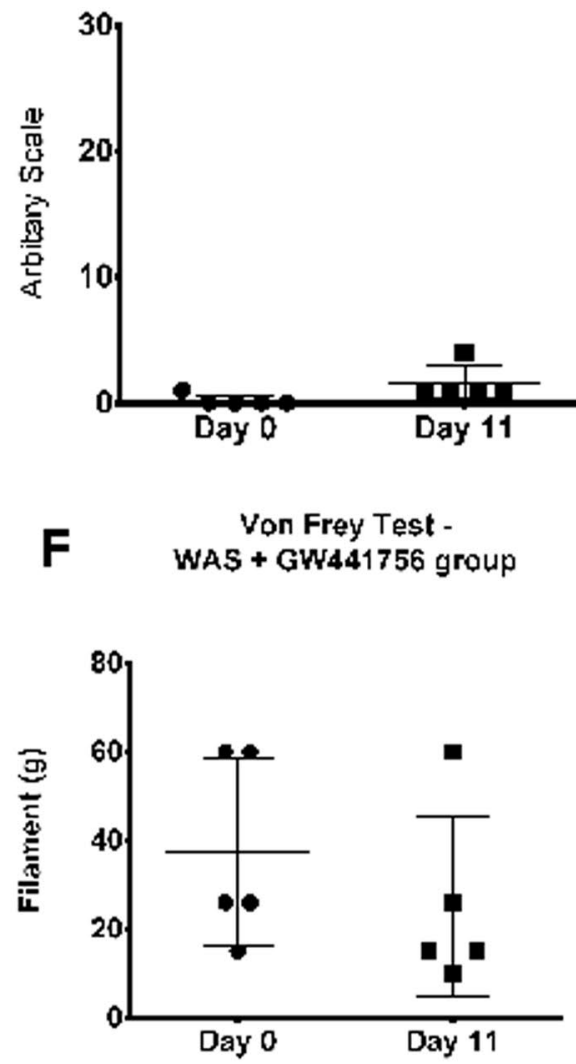

Figure 2. Scatter graph showing pain related behaviour arbitrary values before (day 0) and after (day 11) WAS induction for test A (A) and test B (B). Scatter graph showing pain related behaviour arbitrary values before (day 0) and after (day 11) WAS + Trk A antagonist GW441756 (WAS+ GW441756) administration for test A (C) and test B. (D) Scatter graph showing the lower abdominal mechanical pain threshold using Von Frey filaments before (day 0) and after (day 11) WAS induction (E) and before (day 0) and after (day 11) WAS+ Trk A antagonist GW441756 (WAS + GW441756) administration (F). *p $<0.05$.

In the explant experiments, the culture medium of the non-stimulated bladder strips (control) contained $4.97 \pm 1.06 \mathrm{pg} / \mathrm{ml} . \mathrm{mg}$ bladder strip (Fig. 1C). The stimulation with $1.25 \mathrm{~g} / \mathrm{l}$ of phenylephrine did not induce any change in the NGF released to the media $(4.75 \pm 0.94 \mathrm{pg} / \mathrm{ml} . \mathrm{mg}$ bladder strip; Fig. 3C; Mann-Whitney Test: $\mathrm{p}=0.6571)$.

Evaluation of pain behaviour. The WAS group increase the pain score from day 0 to day 10 , from values of $0.0 \pm 0.0$ to $6.4 \pm 4.6$ in test A (Fig. $2 \mathrm{~A}$; paired t test: $\mathrm{p}=0.0493$ ), and from values of $0.0 \pm 0.0$ to $7.6 \pm 3.6$ in test $\mathrm{B}$ (Fig. 2B; paired t test: test $\mathrm{B}, \mathrm{p}=0.0129)$. In the WAS animals treated daily with $\mathrm{GW} 441756$, a specific TrkA antagonist, the pain score was maintained in baseline values throughout the experiment for both tests. For test A, 


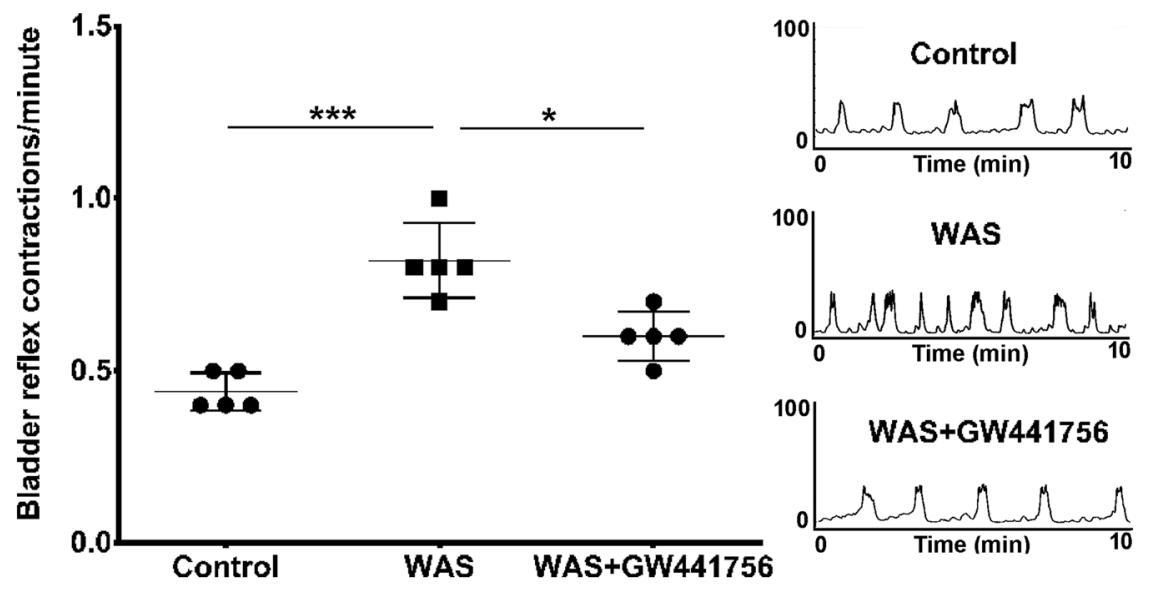

Figure 3. Scatter graph showing the variation of voiding frequency of control animals, animals submitted to WAS and animals submitted to WAS treated with Trk A antagonist GW441756 (WAS+ GW441756) $\left({ }^{*} \mathrm{p}<0.05\right.$; *** $\mathrm{p}<0.001)$. Typical cystometrogram are presented for each group of animals ( $\mathrm{Y}$ axis - Pressure $\left(\mathrm{cm} \mathrm{H}_{2} \mathrm{O}\right), \mathrm{X}$ axis - Time (minutes).

the values were $0.0 \pm 0.0$ at day 0 and $0.2 \pm 0.4$ at day 10 (Fig. $2 \mathrm{C}$; paired $\mathrm{t}$ test: $\mathrm{p}=0.3739$ ). For test $\mathrm{B}$, the values were $0.2 \pm 0.4$ at day 0 and $1.6 \pm 1.2$ at day 10 (Fig. $2 \mathrm{D}$; paired t test: $\mathrm{p}=0.1079$ ).

The mechanical pain threshold of WAS group decrease from day 0 basal value of $40 \pm 24 \mathrm{~g}$ to day 10 value of $6 \pm 2 \mathrm{~g}$ (Fig. $2 \mathrm{E}$; paired t test: $\mathrm{p}=0.0415$ ). The daily administration of GW441756 prevented the decrease in mechanical pain threshold. At day 0 the animals presented abdominal mechanical pain threshold of $37 \pm 19 \mathrm{~g}$ and at day 10 the mechanical pain threshold was $25 \pm 18 \mathrm{~g}$ (Fig. $2 \mathrm{~F}$; paired $\mathrm{t}$ test: $\mathrm{p}=0.2374$ ).

Bladder reflex activity evaluation by cystometry. Sham animals had $0.44 \pm 0.05$ bladder voiding reflex contractions/minute. The animals from WAS group presented $0.82 \pm 0.10$ bladder voiding reflex contractions/minute (Fig. 3; ANOVA followed by Tukey's multiple comparison test: $p=0.0008$ ). The administration of GW441756 decreased bladder reflex contractions to $0.60 \pm 0.0$ (Fig. 3; ANOVA followed by Tukey's multiple comparison test: $\mathrm{p}=0.0186$ compared with WAS group and $\mathrm{p}<0.05$ compared with sham group).

Noradrenaline levels determined by HPLC. The bladder of sham group had $0.21 \pm 0.03$ pmol of noradrenaline $/ \mathrm{mg}$ of bladder homogenate (Fig. 4A). After WAS, animals presented $2.26 \pm 0.26$ pmol of noradrenaline/mg of bladder ( $<<0.001$; Fig. $4 \mathrm{~A}$ ). Plasmatic levels of noradrenaline in the sham and WAS groups were $0.66 \pm 0.35 \mathrm{nmol} / \mathrm{ml}$ and $1.99 \pm 0.64 \mathrm{nmol} / \mathrm{ml}$, respectively $(\mathrm{p}<0.05 ;$ Fig. $4 \mathrm{~B})$.

\section{Discussion}

The most important finding of our study was the marked increase of NGF in the serum after the exposure of naïve female rats to WAS condition. In animals stimulated by WAS test, serum NGF levels increased 114 times, by far exceeding the 3 times increment observed in the urine of the same animals. Among the consequences of the systemic increase of the neurotrophin, a strong sensitization of the nociceptors throughout the body of the animals may have occurred, explaining the intense pain behaviour, the decrease of mechanical pain threshold in lower abdominal and the duplication of the number of bladder voiding reflex contractions. All this changes were dependent upon the activation of the TrkA receptors, the high-affinity receptors for NGF and consequent nociceptors sensitization. In fact, the blockade of TrkA with its specific antagonist GW441756 prevented the manifestations seen in non-treated WAS animals.

The levels of NGF in the urine of WAS animals was much more modest than in serum. These experimental findings agree with observations in BPS/IC patients in whom the mean NGF serum levels were $3.48 \mathrm{pg} / \mathrm{mL}$ while the mean urinary levels, corrected for creatinine, were only $0.69 \mathrm{pg} / \mathrm{mg}^{14}$. These observations raise the hypothesis that the source of the neurotrophin may be other than the bladder wall. The marked increase of serum NGF may in fact reflect a systemic increase of the neurotrophin rather than a bladder synthesis and its posterior release into the serum and urine. It would be difficult to support that the bladder, able to increase the urinary levels by 3 times, were able to increase the serum levels by 114 times. The appearance of NGF in the urine could, in this hypothesis, result of its diffusion from bladder blood vessels into the urine. In addition, due to its low molecular weight (13.2 kDa, five times less than albumin molecular weight), NGF can appear in urine after filtration in renal glomeruli. Moreover, the fact that $24 \mathrm{~h}$ stimulation with phenylephrine intended to mimic the stressful conditions in culture experiments did not change NGF levels in culture medium containing our whole bladder strips reinforces the hypothesis that the serum NGF detected in the blood of WAS rats was synthetized outside the bladder. It may be recalled that the time of exposure to phenylephrine in culture experiments were shown to be sufficient for catecholamines to induce a robust release of NGF from non-neuronal cells into culture medium ${ }^{15}$.

If the above experimental and clinical observations may not support the bladder tissue as the main source for the increased serum levels of NGF, it might be important to discuss other possible origins for the neurotrophin. Mast cells have been pointed out as another important source of $\mathrm{NGF}^{16}$. Mast cells have been shown to be 
A

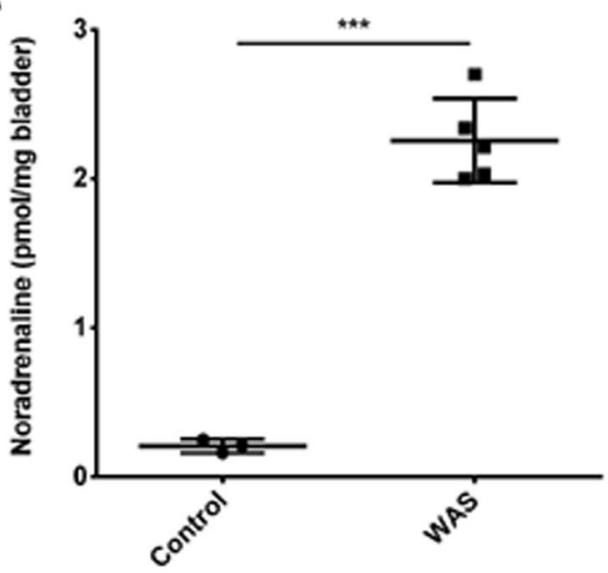

B

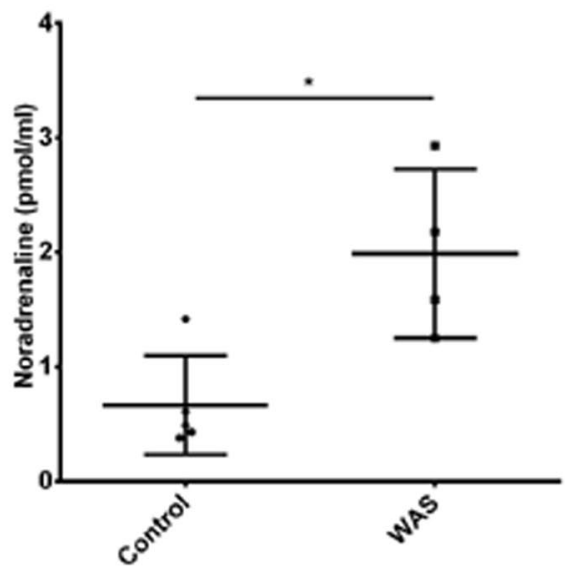

Figure 4. Scatter graph showing the noradrenaline levels in (A) the bladder (pmol/mg) and (B) serum (pmol/ $\mathrm{ml}$ ) of animals from control and WAS groups. $* \mathrm{p}<0.05 ; * * \mathrm{p}<0.001$.

increased in the bladder tissue of BPS/IC patients which course with a heavy inflammation of the organ, but not in other organs of BPS/IC patients. Therefore, mast cells are an unlikely source for the systemic increase of NGF in WAS rats. Other cells that may synthetize and release NGF are fibroblasts. These cells, in in vitro experiments, were shown to release NGF into the culture media though a process that, interestingly, was stimulated by catecholamines ${ }^{15}$. These findings agree well with our present findings. The rat model of WAS courses with increased levels of noradrenaline in the serum and in the bladder wall.

The administration of silodosin, an alpha $1 \mathrm{~A}$ adrenoceptor antagonist was shown to prevent NGF increase, to supress pain behaviour, to normalize mechanical pain threshold and bladder reflex voiding contractions in WAS rats. It may be recalled that in previous studies, we showed that chronic exogenous administration of an adrenergic agent, phenylephrine, to intact rats induced a robust spinal cord expression of c-fos, a surrogate marker of pain, after innocuous bladder stimulation. Spinal c-fos expression was prevented by the administration of alpha1A adrenoceptor antagonists ${ }^{13}$.

Among the most frequently used models of chronic bladder pain in rodents are the ones in which the urothelium is severely damaged with cyclophosphamide or protamine. In these models an intense pan-mural inflammation swiftly develops in the bladder. In these models a rapid increase of NGF and other inflammatory mediators is expected to occur ${ }^{17}$. Unfortunately, in these models the serum NGF was not systematically measured for comparison with the urinary levels. However, taking in consideration the nature of the models it is expectable that the results might be substantially different from those obtained in the WAS model used in the present study. Comparisons among rodent models of BPS/IC are lacking but are utterly necessary in order to compare the outcomes reported.

The implications of our observations are multiple. Serum NGF may become an important biomarker to phenotype BPS/IC patients. Being a disease which has no defined etiology yet, grouping patients with similar characteristics may foster the process of discovery. In fact, the etiopathogenesis is better recognized in homogeneous than in heterogeneous groups. NGF measurement in the blood may help to identify patients that respond better to humanized antibodies presently under development to sequester an excess of NGF. Most likely, patients with high levels of the neurotrophin will be the best responders. Recent studies showed that women with BPS/IC and associated non-urological somatic syndromes were more likely to experience significant pain reduction with antibodies anti-NGF ${ }^{2,18}$. Those characteristics may well be a consequence of high NGF levels in the blood leading to a systemic sensitization of somatic and non-bladder visceral nociceptors. It is important to mention that in a small cohort of 28 BPS/IC patients, an elevated serum NGF was detected in only 17 (60\%) of them, potentially reinforcing the importance of NGF as a biomarker for patient phenotyping ${ }^{19}$. The present study also suggests that TrkA antagonist may be used in lieu of anti-NGF antibodies. In addition to the obvious facility in its administration (NGF antibodies require sub-cutaneous injections while TrkA antagonists are expected to work easily by oral route) TrkA antagonists may also be useful by intravesical route, as long as small molecules that cross the urothelium can be produced.

The capacity of silodosin to prevent the increase in plasmatic NGF and at the same time to avoid the development of pain behaviour, bladder reflex hyperactivity and decrease in abdominal mechanical pain threshold in animals exposed to WAS may suggest that this alpha blocker can be useful in the pain management of some BPS/ IC women, in particular those showing evident signs of stress and non-urological symptoms. This drug has been extensively investigated in men with lower urinary symptoms associated with enlarged prostates and did not show any serious adverse events during its administration ${ }^{20}$. Therefore, a trial with silodosin in women with BPS and signs of stress may be of interest.

The activation of TrkA receptors influences the expression of other pain receptors ${ }^{21}$. A previous study showed that NGF-induced bladder overactivity and bladder noxious input was depending on the interaction of NGF with TRPV $1^{22}$. In fact, NGF had no effects of TRPV1 KO mice ${ }^{22}$. TRPV1 synthesis and transport to the periphery is stimulated by $\mathrm{NGF}^{23}$. If TRPV1 represents a bottleneck pathway in bladder pathologies associated with NGF 
up-regulation, TRPV1 antagonists may demonstrate therapeutic relevance in BPS/IC patients as well. In fact, earliest TRPV1 antagonists available were shown to reduce bladder pain and bladder reflex activity in rodent models of chemical bladder inflammation ${ }^{24}$.

In conclusion, our exploratory study demonstrates that chronic stress causes an increase of NGF in the serum and in the urine. Being the levels in the former much higher than those in the urine, it is unlikely that the blood NGF has its origin only in the bladder. Identification of extra-bladder tissues able to synthetize and release NGF in patients with BPS/IC is therefore necessary. Finally, NGF sequestration or TrkA antagonists is supported as an important therapy for BPS/IC.

\section{Methods}

This work was approved by the animal ethical committee "ORBEA - Organismo Responsável pelo Bem-Estar dos Animais" from our institution as well as by the national animal ethical committee DGAV. All experiments were performed in accordance with relevant guidelines and regulations.

Animal model. A group of five adult female Wistar rats (200-225g) obtained from our animal facility were submitted to Water Avoidance Stress (WAS) test (WAS group), as recently described. Briefly, animals were placed on pedestals (cylinder $\left.52 \pi \times 14 \mathrm{~cm}^{3}\right)$ in the centre of cages $\left(26.6 \times 42.5 \times 18.0 \mathrm{~cm}^{3}\right)$, filled with water at room temperature, for $1 \mathrm{~h} /$ day, for ten consecutive days. The experiments were performed between 10:00 a.m. and 12:00 a.m. to minimize circadian effects. The experiments were performed under red light as animals were housed in inverted light cycle.

Another group of five adult female Wistar rats were submitted to WAS while orally received the TrkA antagonist GW441756, $58 \mu \mathrm{g} / \mathrm{kg} /$ day (Biotechne).

Another group of four adult female Wistar rats were submitted to WAS while orally received the alpha $1 \mathrm{~A}$ adrenoceptor silosodin at $0.2 \mathrm{mg} / \mathrm{kg}$.day (commercial formulation available from Recordati Urorec ${ }^{\circledR}$ ). This group was only used to collect blood for the determination of NGF levels.

Another group of five adult female Wistar rats were placed on the pedestals in waterless cages (Sham group). This group was only used as control group for cystometries and for NGF determination in serum and urine.

Another group of four adult female Wistar rats was used only for explant cultures experiments (intact group).

Bladder explant culture experiments. The bladders of intact rats were collected, and bladder strips were cut and left in a urothelial cell culture medium (Cat No 4321; ScienCell), in culture medium with $1.25 \mathrm{~g} / \mathrm{l}$ of phenylephrine (P6126, Sigma) or in culture medium with $1.25 \mathrm{~g} / \mathrm{l}$ of phenylephrine and $0.1 \mathrm{~g} / \mathrm{l}$ of the alpha $1 \mathrm{~A}$ adrenoceptor blocker silodosin (commercial formulation available from Recordati Urorec ${ }^{\circledR}$ ). After $24 \mathrm{~h}$, the medium was collected to measure NGF by ELISA.

NGF levels evaluation by ELISA. Plasma, urine and culture media were used to analyse NGF levels by ELISA, following the manufactures instruction (ELH-BNGF RayBio Human beta-NGF Kit; minimum sensitivity is $14 \mathrm{pg} / \mathrm{ml}$ ). The manufacture informed that this ELISA kit shows no cross-reactivity with the following cytokines tested: human Angiogenin, BDNF, BLC, ENA-78, FGF- 4, IL-1 alpha, IL-1 beta, IL-2, IL-3, IL-4, IL-5, IL-6, IL-7, IL-8, IL-9, IL-10, IL-11, IL-12 p70, IL-12 p40, IL-13, IL-15, I-309, IP-10, G-CSF, GM-CSF, IFN-gamma, Leptin (OB), MCP-1, MCP-3, MDC, MIP-1 alpha, MIP-1 beta, MIP-1 delta, MMP-1, - 2, -3, -10, PARC, RANTES, SCF, TARC, TGF-beta, TIMP-1, TIMP-2, TNF-alpha, TNF-beta, TPO, VEGF. No other proteins were tested in the present work.

Briefly, soluble NGF from samples bind to anti-NGF coated plates. Captured NGF was recognized by a biotinylated secondary antibody that was combined with HRP conjugated streptavidin. The chromogenic substrate 3,3,5,5'-tetramethylbenzidine was added for colour development and sulphuric acid was used to stop the reaction. The amount of NGF in the sample was measured at $450 \mathrm{~nm}$ with a Synergy HT Microplate Reader (BioTek Instruments, USA). All samples and standards were run in duplicate and values were averaged against a standard curve generated with known amounts of NGF.

Noradrenaline evaluation. Bladder and urine were used to analyse noradrenaline levels by HPLC. Urines were acidified with $\mathrm{HCl} 6 \mathrm{M}$ and immediately stored at $-20^{\circ} \mathrm{C}$, until urine of all groups was gathered and then transferred to $-80^{\circ} \mathrm{C}$. Noradrenaline levels were determined by HPLC with electrochemical detection, as previously described ${ }^{25}$. Briefly, aliquots of urine were added to $50 \mathrm{mg}$ alumina (to adsorb noradrenaline) and the $\mathrm{pH}$ was adjusted to $\mathrm{pH} 8.3-8.6$ with TRIS buffer. Then noradrenaline was eluted with $200 \mu \mathrm{l}$ of $0.2-\mathrm{M}$ perchloric acid on Costar Spin-X microfilters, and $50 \mu \mathrm{l}$ of the eluate was injected into an HPLC (Gilson Medical Electronics, Villiers le Bel,France). The lower limit of detection of noradrenaline was $350 \mathrm{fmol}$.

Evaluation of pain behaviour. After an initial period of habituation (repeated 5 days before day 0 ) to the experimental environment and to handler, visceral pain behavioural tests and lower abdomen pain were evaluated on WAS and WAS + GW441756 groups, using a mechanical hyperalgesia test previously described. Briefly, to evaluate visceral pain behaviour, two test were performed. In test A, animal breathing rate, eyes aperture and body posture using were analysed at day 0 (control) and at day 10 . An arbitrary $0-10$ scale was used for each parameter. For the breathing rate, every decrease of 10 cycle/minute compared with day 1 record was scored 1 . For the eye aperture test, the following scale was used: 0 for complete opening, 2 for intermediate opening, 5 for half-closed, 7 for intermediate opening and 10 for complete closing. For body posture, if animals presented rounded-back with body aligned or motionlessness the score was 10 . Otherwise the score was 0 . In test $\mathrm{B}$, the following parameters were scored: normal 0 , piloerection 1 , strong piloerection 2 , laboured breathing 3 , licking the abdomen 4 and stretching and contractions of the abdomen 5 . 
To evaluate mechanical hyperalgesia, at day 0 and day 10 , animals were placed in individual chambers with a wire mesh floor and allowed to acclimate. Then, a series of Von Frey monofilaments (rated from 4 to $60 \mathrm{~g}$ ) were applied perpendicularly in the lower abdomen, with enough strength to cause the monofilament to slightly bend. Each monofilament was tested five times, with $5 \mathrm{~s}$ interval between each application. If there was abdomen retraction or the animal jump in at least three of the five filament applications, it was considered a positive response. In case of no response to the filament, the next-stronger monofilament was applied within an interval of $30 \mathrm{~s}$.

Bladder reflex activity evaluation by cystometry. At day 11, after urine collection from sham and WAS groups, animals from sham, WAS and WAS + GW441756 groups were anaesthetised with urethane (1.2 g/ $\mathrm{kg}$ body weight) laid on a heating pad with a rectal probe inserted to measure body temperature. The bladder was then exposed through a low abdominal incision. A 20-gauge needle was inserted in the bladder dome and saline was infused $(6 \mathrm{ml} / \mathrm{h})$. After a stabilization period of $30 \mathrm{~min}$, the cystometrograms were recorded for $10 \mathrm{~min}$. Cystometrograms were analysed, and bladder reflex activity was expressed as the number of bladder contractions per minute. After the cystometry, blood was collected from the heart from sham, WAS and WAS + GW441756 groups, using a needle coated with heparin and centrifuged at $1000 \mathrm{~g}$ for 15 minutes and plasma was collected and stored at $-20^{\circ} \mathrm{C}$ to measure NGF by ELISA and noradrenaline by HPLC. Blood was also collected from animals of WAS + silodosin group to measure NGF by ELISA. Afterwards, animals were euthanized.

Statistical analysis. Pain behaviour score, mechanical pain threshold, bladder reflex activity and NGF values are represented as mean \pm standard deviation. Statistical analysis was performed using Graph Pad Prism 7. When comparing two groups, significance was estimated using Mann-Whitney Test. When comparing more than two groups, significance was estimated using ANOVA followed by Tukey's multiple comparisons test, as all data showed to follow a normal distribution and to minimize Type I errors (false positive).

\section{References}

1. McMahon, S. B., Bennett, D. L., Priestley, J. V. \& Shelton, D. L. The biological effects of endogenous nerve growth factor on adult sensory neurons revealed by a trkA-IgG fusion molecule. Nat. Med. 1, 774-780 (1995).

2. Chen, W. et al. Elevated level of nerve growth factor in the bladder pain syndrome/interstitial cystitis: a meta-analysis. Springerplus 5, 1072 (2016).

3. Kim, S. W. et al. Urinary nerve growth factor correlates with the severity of urgency and pain. Int. Urogynecol. J. 25, 1561-1567 (2014).

4. Pinto, R. et al. Trigonal injection of botulinum toxin A in patients with refractory bladder pain syndrome/interstitial cystitis. Eur Urol 58, 360-365 (2010).

5. Tonyali, S. et al. Urine nerve growth factor (NGF) level, bladder nerve staining and symptom/problem scores in patients with interstitial cystitis. Adv. Clin. Exp. Med. 27, 159-163 (2018).

6. Liu, H. T., Tyagi, P., Chancellor, M. B. \& Kuo, H. C. Urinary nerve growth factor level is increased in patients with interstitial cystitis/ bladder pain syndrome and decreased in responders to treatment. BJU Int 104, 1476-1481 (2009).

7. Jiang, Y. H., Peng, C. H., Liu, H. T. \& Kuo, H. C. Increased pro-inflammatory cytokines, C-reactive protein and nerve growth factor expressions in serum of patients with interstitial cystitis/bladder pain syndrome. PLoS One 8, e76779 (2013).

8. Nickel, J. C. et al. Tanezumab Reduces Pain in Women with Interstitial Cystitis/Bladder Pain Syndrome and Patients with Nonurological Associated Somatic Syndromes. J Urol 195, 942-948 (2016).

9. Tanner, R., Chambers, P., Khadra, M. H. \& Gillespie, J. I. The production of nerve growth factor by human bladder smooth muscle cells in vivo and in vitro. BJU Int. 85, 1115-1119 (2000).

10. Girard, B. M., Malley, S. E. \& Vizzard, M. A. Neurotrophin/receptor expression in urinary bladder of mice with overexpression of NGF in urothelium. Am. J. Physiol. Renal Physiol. 300, F345-55 (2011).

11. Twiss, C. et al. Increased startle responses in interstitial cystitis: evidence for central hyperresponsiveness to visceral related threat. J Urol 181, 2127-2133 (2009).

12. Charrua, A. et al. Can the adrenergic system be implicated in the pathophysiology of bladder pain syndrome/interstitial cystitis? A clinical and experimental study. Neurourol. Urodyn. 34 (2015).

13. Matos, R., Cordeiro, J. M., Igawa, Y., Cruz, F. \& Charrua, A. Bladder Pain In Bps/Ic May Result From An Exaggerated Response Of Trpv1 And Enhanced Urothelial Atp Release Induced By Chronic Adrenergic Overactivity. Neurourol. Urodyn. 34, S341-S342 (2015).

14. Liu, H. T. \& Kuo, H. C. Increased urine and serum nerve growth factor levels in interstitial cystitis suggest chronic inflammation is involved in the pathogenesis of disease. PLoS One 7, e44687 (2012).

15. Furukawa, Y. et al. Catecholamines increase nerve growth factor mRNA content in both mouse astroglial cells and fibroblast cells. FEBS Lett. 247, 463-467 (1989).

16. Skaper, S. D. Nerve growth factor: a neuroimmune crosstalk mediator for all seasons. Immunology 151, 1-15 (2017).

17. Vizzard, M. A. Changes in urinary bladder neurotrophic factor mRNA and NGF protein following urinary bladder dysfunction. Exp. Neurol. 161, 273-284 (2000).

18. Chuang, Y.-C., Chermansky, C., Kashyap, M. \& Tyagi, P. Investigational drugs for bladder pain syndrome (BPS)/interstitial cystitis (IC). Expert Opin. Investig. Drugs 25, 521-529 (2016).

19. Kuo, H. C. Potential urine and serum biomarkers for patients with bladder pain syndrome/interstitial cystitis. Int J Urol 21(Suppl 1), 34-41 (2014).

20. Villa, L. et al. Silodosin: An Update on Efficacy, Safety and Clinical Indications in Urology. Adv. Ther. 36, 1-18 (2019).

21. Ochodnicky, P., Cruz, C. D., Yoshimura, N. \& Cruz, F. Neurotrophins as regulators of urinary bladder function. Nat. Rev. Urol. 9 , 628-637 (2012)

22. Frias, B. et al. Transient receptor potential vanilloid 1 mediates nerve growth factor-induced bladder hyperactivity and noxious input. BJU Int. $110(2012)$

23. Ji, R.-R., Samad, T. A., Jin, S.-X., Schmoll, R. \& Woolf, C. J. p38 MAPK activation by NGF in primary sensory neurons after inflammation increases TRPV1 levels and maintains heat hyperalgesia. Neuron 36, 57-68 (2002).

24. Charrua, A. et al. GRC-6211, a New Oral Specific TRPV1 Antagonist, Decreases Bladder Overactivity and Noxious Bladder Input in Cystitis Animal Models. J. Urol. 181, (2009).

25. Soares-da-Silva, P., Pestana, M., Vieira-Coelho, M. A., Fernandes, M. H. \& Albino-Teixeira, A. Assessment of renal dopaminergic system activity in the nitric oxide-deprived hypertensive rat model. Br J Pharmacol 114, 1403-1413 (1995). 


\section{Acknowledgements}

The statements and opinions presented here reflect the author's view and neither IMI nor the European Union, EFPIA, or any Associated Partners are responsible for any use that may be made of the information contained therein. This project has received funding from the Innovative Medicines Initiative 2 Joint Undertaking under grant agreement No [777500]. This Joint Undertaking receives support from the European Union's Horizon 2020 research and innovation programme and EFPIA. www.imi.europa.eu. www.imi-paincare.eu. This work was partially supported by PFD Research Foundation and ICA IC/PBS Research Grant.

\section{Author Contributions}

Bruno Dias and Ana Charrua performed experiments. Paula Serrão performed noradrenaline quantification by HPLC. Francisco Cruz and Ana Charrua design the experiments. All authors contributed to manuscript writing.

\section{Additional Information}

Competing Interests: Bruno Dias, Paula Serrão e Ana Charrua have no conflict of interests. Francisco Cruz is consultant and investigator for Astellas and Recordati.

Publisher's note Springer Nature remains neutral with regard to jurisdictional claims in published maps and institutional affiliations.

(c) (i) Open Access This article is licensed under a Creative Commons Attribution 4.0 International

License, which permits use, sharing, adaptation, distribution and reproduction in any medium or format, as long as you give appropriate credit to the original author(s) and the source, provide a link to the Creative Commons license, and indicate if changes were made. The images or other third party material in this article are included in the article's Creative Commons license, unless indicated otherwise in a credit line to the material. If material is not included in the article's Creative Commons license and your intended use is not permitted by statutory regulation or exceeds the permitted use, you will need to obtain permission directly from the copyright holder. To view a copy of this license, visit http://creativecommons.org/licenses/by/4.0/.

(C) The Author(s) 2019 